\title{
HARMONIC FUNCTIONS HAVING NO TANGENTIAL LIMITS
}

\author{
HIROAKI AIKAWA
}

(Communicated by Irwin Kra)

\begin{abstract}
Let $C_{0}$ be a tangential curve in $D=\{|z|<1\}$ which ends at 1 and let $C_{\theta}$ be its rotation about the origin through an angle $\theta$. We construct a bounded harmonic function in $D$ which fails to have limits along $C_{\theta}$ for all $\theta, 0 \leq \theta \leq 2 \pi$
\end{abstract}

\section{INTRODUCTION}

The classical Fatou theorem asserts that if $f$ is a bounded holomorphic function in the unit disc $D=\{|z|<1\}$, then $f$ has nontangential boundary values at almost every point on the unit circle $\partial D=\{|z|=1\}$. The Fatou theorem has been generalized in several directions; a bounded (or more generally, positive) harmonic function in $D$ has nontangential boundary values at almost every point on $\partial D$. In 1926, Littlewood [3] showed that the Fatou theorem is best possible in the following sense: Let $C_{0}$ be an arbitrary tangential curve in $D$ which ends at $z=1$ and let $C_{\theta}$ be the curve $C_{0}$ rotated about the origin through an angle $\theta$, so that $C_{\theta}$ touches $\partial D$ internally at $e^{i \theta}$.

Theorem A. There exists a bounded harmonic function $h$ in $D$ such that

$$
\lim _{|z| \rightarrow 1, z \in C_{\theta}} h(z)
$$

does not exist for almost all $\theta, 0 \leq \theta \leq 2 \pi$. In particular, there exists $a$ bounded holomorphic function $f$ in $D$ such that

$$
\lim _{|z| \rightarrow 1, z \in C_{\theta}} f(z)
$$

does not exist for almost all $\theta, 0 \leq \theta \leq 2 \pi$.

The second half of Theorem A, in fact, readily follows from the first; if $h^{*}$ is a harmonic conjugate function of $h$, then $\exp \left(h+i h^{*}\right)$ is a bounded outer function in $D$ with the property holding for almost all $\theta$. In 1957, Lohwater and Piranian [4] (see also Collingwood and Lohwater [2; Theorem 2.22]) improved the second half of Theorem $A$ by giving the next theorem.

Received by the editors September 26, 1988 and, in revised form, April 11, 1989.

1980 Mathematics Subject Classification (1985 Revision). Primary 31A20, 30D40.

Key words and phrases. Fatou theorem, harmonic functions, tangential limits. 
Theorem B. There exists a Blaschke product $B$ in $D$ such that

$$
\lim _{|z| \rightarrow 1, z \in C_{\theta}} B(z)
$$

does not exist for all $\theta, 0 \leq \theta \leq 2 \pi$.

Since the logarithm of the modulus of a Blaschke product is a Green potential of a measure distributed on the zeros of the Blaschke product, we may, from the potential theoretical point of view, regard Theorem B as the existence of a Green potential which fails to have limits along $C_{\theta}$ for all $\theta, 0 \leq \theta \leq 2 \pi$. The existence of a bounded harmonic function with the property holding for all $\theta$, however, seems to be unknown. In 1980, Barth (see [1; p. 551]) raised a somewhat weaker question. Does there exist a positive harmonic function which fails to have limits along $C_{\theta}$ for all $\theta, 0 \leq \theta \leq 2 \pi$ ?

The purpose of this paper is to show

Theorem. There exists a bounded harmonic function $h$ in $D$ such that

$$
\lim _{|z| \rightarrow 1, z \in C_{\theta}} h(z)
$$

does not exist for all $\theta, 0 \leq \theta \leq 2 \pi$. In particular, if $h^{*}$ is a harmonic conjugate function of $h$, then $\exp \left(h+i h^{*}\right)$ is a bounded outer function in $D$ which fails to have limits along $C_{\theta}$ for all $\theta, 0 \leq \theta \leq 2 \pi$.

Obviously, the above theorem automatically answers Barth's question in the affirmative. However, we note that Barth's question itself can be solved more easily. We shall, in fact, find a positive unbounded harmonic function with the required property (see Remark in $\S 3$ ).

\section{LEMMAS}

For an integrable function $f$ on $[0,2 \pi]$ we let

$$
\operatorname{PI}(f, z)=\frac{1}{2 \pi} \int_{0}^{2 \pi} \frac{1-|z|^{2}}{\left|z-e^{i \varphi}\right|^{2}} f(\varphi) d \varphi
$$

be the Poisson integral of $f$. We identify the left end 0 and the right end $2 \pi$ of the interval $[0,2 \pi]$. If an interval $\left[\theta_{1}, \theta_{2}\right]$ is not contained in $[0,2 \pi]$, we replace it by

$$
\left\{\Theta=\theta-2 n \pi ; \theta_{1} \leq \theta \leq \theta_{2} \text { and } n=\left[\frac{\theta}{2 \pi}\right]\right\} .
$$

Here [.] denotes the greatest integer function. We shall continue to refer to sets defined by $(*)$ as intervals.

Let us begin with some elementary estimates of the Poisson integrals.

Lemma 1. Let $m>\pi / 2,0<c<1$ and $\eta \in[0,2 \pi]$. Suppose $f$ is measurable on $[0,2 \pi],|f| \leq 1$ on $[0,2 \pi]$ and $f(\varphi)=0$ for $|\varphi-\eta|<m c$. Then

$$
\left|\mathrm{PI}\left(f, r e^{i \eta}\right)\right| \leq \frac{2}{\pi}\left(\frac{2}{\pi}-\frac{1}{m}\right)^{-2} m^{-1} \quad \text { for } 1-c \leq r<1 .
$$


Proof. Let $1-c \leq r<1$. We observe that if $m c \leq|\varphi-\eta| \leq \pi$, then $\left|r e^{i \eta}-e^{i \varphi}\right| \geq\left|e^{i \varphi}-e^{i \eta}\right|-(1-r) \geq 2\left|\sin \frac{\varphi-\eta}{2}\right|-\frac{1}{m}|\varphi-\eta| \geq\left(\frac{2}{\pi}-\frac{1}{m}\right)|\varphi-\eta|$.

Hence

$$
\left|\mathrm{PI}\left(f, r e^{i \eta}\right)\right| \leq \frac{1-r^{2}}{2 \pi}\left(\frac{2}{\pi}-\frac{1}{m}\right)^{-2} \int_{|\varphi-\eta| \geq m c} \frac{d \varphi}{|\varphi-\eta|^{2}},
$$

which implies the desired inequality.

Lemma 2 (cf. [3; Lemma 2]). Let $m_{1}>\pi / 2$ be a constant such that

$$
\frac{2}{\pi}\left(\frac{2}{\pi}-\frac{1}{m_{1}}\right)^{-2} m_{1}^{-1} \leq \frac{1}{4}
$$

Let $0<c<1$ and $\eta \in[0,2 \pi]$. Suppose $f$ is measurable on $[0,2 \pi],|f| \leq 1$ on $[0,2 \pi]$ and $f(\varphi)=1$ for $|\varphi-\eta|<m_{1} c$. Then

$$
\operatorname{PI}\left(f, r e^{i \eta}\right) \geq \frac{1}{2} \quad \text { for } 1-c \leq r<1 .
$$

Proof. We write $\mathrm{PI}\left(f, r e^{i \eta}\right)=1+2 \mathrm{PI}\left(\frac{1}{2}(f-1), r e^{i \eta}\right)$ and apply Lemma 1 to $\frac{1}{2}(f-1)$ to obtain

$$
\mathrm{PI}\left(f, r e^{i \eta}\right) \geq 1-2 \cdot \frac{2}{\pi}\left(\frac{2}{\pi}-\frac{1}{m_{1}}\right)^{-2} m_{1}^{-1} \geq \frac{1}{2} .
$$

Lemma 3. Let $0<\varepsilon<\frac{1}{4}$ and $0<c<1$. Suppose $f$ is measurable on $[0,2 \pi]$, $|f| \leq 1$ on $[0,2 \pi]$ and

$$
c^{-1} \int_{|\varphi-\eta|<c}|f(\varphi)| d \varphi \leq \varepsilon
$$

for all $\eta \in[0,2 \pi]$. Then

$$
\sup _{|z| \leq 1-c}|\operatorname{PI}(f, z)| \leq m_{2} \sqrt{\varepsilon},
$$

where $m_{2}=2 / \pi+(2 / \pi)(2 / \pi-1 / 2)^{-2}$.

Proof. Let $r=1-c$ and take an arbitrary point $r e^{i \eta}$ on the circle $\{|z|=r\}$. We decompose $f$ into $f_{1}+f_{2}$, where $f_{1}=f \chi_{|\varphi-\eta|<c / \sqrt{\varepsilon}}$. Then

$$
\begin{aligned}
\left|\mathrm{PI}\left(f_{1}, r e^{i \eta}\right)\right| & \leq \frac{1}{2 \pi} \int_{|\varphi-\eta|<c / \sqrt{\varepsilon}} \frac{1-r^{2}}{(r-1)^{2}}|f(\varphi)| d \varphi \\
& \leq \frac{1}{2 \pi} \cdot \frac{2}{c} \cdot c \varepsilon(1+[1 / \sqrt{\varepsilon}]) \leq \frac{2}{\pi} \sqrt{\varepsilon},
\end{aligned}
$$

where $[1 / \sqrt{\varepsilon}]$ stands for the greatest integer not greater than $1 / \sqrt{\varepsilon}$. Here the second inequality follows from (1) and the fact that the interval $\{|\varphi-\eta|<c / \sqrt{\varepsilon}\}$ 
of length $2 c / \sqrt{\varepsilon}$ is covered by at most $1+[1 / \sqrt{\varepsilon}]$ intervals of length $2 c$. Applying Lemma 1 to $f=f_{2}$ and $m=1 / \sqrt{\varepsilon}$, we obtain

$$
\left|\mathrm{PI}\left(f_{2}, r e^{i \eta}\right)\right| \leq \frac{2}{\pi}\left(\frac{2}{\pi}-\sqrt{\varepsilon}\right)^{-2} \sqrt{\varepsilon} \leq \frac{2}{\pi}\left(\frac{2}{\pi}-\frac{1}{2}\right)^{-2} \sqrt{\varepsilon} .
$$

Therefore $|\operatorname{PI}(f, z)| \leq m_{2} \sqrt{\varepsilon}$ on the circle $\{|z|=1-c\}$, and hence the maximum principle proves the lemma.

We define the set valued mapping $T$ from $D$ to $[0,2 \pi]$ by

$$
T z=\left\{\theta ; z \in C_{\theta}\right\} .
$$

If none of $C_{\theta}$ contains $z$, then $T z=\varnothing$. Let $M$ be a subset of $D$. We observe that $T(M)=[0,2 \pi]$ if and only if every curve $C_{\theta}, 0 \leq \theta \leq 2 \pi$, meets the set $M$. Let $\gamma$ be a subcurve of $C_{0}$ and let $\gamma_{\theta}$ be its rotation about the origin through $\theta$. Therefore $\gamma=\gamma_{0}$. We define the set valued mapping $T_{\gamma}$ by

$$
T_{\gamma} z=\left\{\theta ; z \in \gamma_{\theta}\right\} \text {. }
$$

Obviously, $T_{\gamma} z \subset T z$. We denote by $\gamma^{*}$ the radial projection of $\gamma$ onto $\partial D$. It follows from the connectedness of $\gamma$ that $\gamma^{*}$ is a circular interval on $\partial D$ or a singleton set. If $\gamma$ contains both the end points, then $\gamma^{*}$ is a closed circular interval or a singleton set. We denote by $l\left(\gamma^{*}\right)$ the length of $\gamma^{*}$. Note that the curve $\gamma$ itself may not be rectifiable.

Lemma 4. Let $\gamma$ be a subcurve of $C_{0}$ connecting $a e^{i \alpha}$ and $b e^{i \beta}, 0<a<b \leq 1$, such that if $z \in \gamma$, then $a \leq|z| \leq b$. Put $M(\eta)=\left\{r e^{i \eta} ; a \leq r \leq b\right\}$. Then $T_{\gamma}(M(\eta))$ is a closed interval of length $l\left(\gamma^{*}\right)$. More precisely, if $\gamma^{*}=\left\{e^{i \theta} ; \theta_{1} \leq\right.$ $\left.\theta \leq \theta_{2}\right\}$, then $T_{\gamma}(M(\eta))=\left[\eta-\theta_{2}, \eta-\theta_{1}\right]$.

Proof. Suppose that $\gamma^{*}=\left\{e^{i \theta} ; \theta_{1} \leq \theta \leq \theta_{2}\right\}$. For each $\theta \in\left[\theta_{1}, \theta_{2}\right]$ we find $r_{\theta}, a \leq r_{\theta} \leq b$, such that $r_{\theta} e^{i \theta} \in \gamma$, or equivalently $r_{\theta} e^{i \eta} \in \gamma_{\eta-\theta}$. Hence $\eta-\theta \in T_{y}(M(\eta))$, so that $\left[\eta-\theta_{2}, \eta-\theta_{1}\right] \subset T_{\gamma}(M(\eta))$. Conversely, if $\theta \in T_{\gamma}(M(\eta))$, then there is $r_{\theta}, a \leq r_{\theta} \leq b$, such that $r_{\theta} e^{i \eta} \in \gamma_{\theta}$. Hence $r_{\theta} e^{i(\eta-\theta)} \in \gamma$, so that $\theta_{1} \leq \eta-\theta \leq \theta_{2}$, or equivalently $\theta \in\left[\eta-\theta_{2}, \eta-\theta_{1}\right]$. Therefore $T_{y}(M(\eta)) \subset\left[\eta-\theta_{2}, \eta-\theta_{1}\right]$.

Since $C_{0}$ is tangential, it follows that $C_{0}$ eventually lies outside any Stoltz region with vertex at the point 1 , so that

$$
\frac{l\left(C_{0}(z)^{*}\right)}{1-|z|} \rightarrow \infty
$$

as $z \in C_{0}$ tends to 1 , where $C_{0}(z)$ is the subcurve of $C_{0}$ that connects the points $z$ and 1 . We have from this fact

Lemma 5. For each $m>1$ we can choose a sequence of subcurves $\gamma_{j}$ of $C_{0}$ with the following properties:

(i) $\gamma_{j}$ connects $a_{j} e^{i \chi_{j}}$ and $b_{j} e^{i \beta_{j}}$. 
(ii) If $z \in \gamma_{j}$, then $a_{j} \leq|z| \leq b_{j}$.

(iii) $l\left(\gamma_{j}^{*}\right)>j\left(1-a_{j}\right)$.

(iv) $0<1-b_{j}<1-a_{j}<\left(1-b_{j-1}\right) / m<\left(1-a_{j-1}\right) / m$.

Proof. We shall choose $\gamma_{j}$ inductively. Assume we have already gotten $\gamma_{0}, \ldots$, $\gamma_{j-1}$ with the properties (i)-(iv). By the observation before the lemma we can take $a_{j}$ such that

$$
0<1-a_{j}<\left(1-b_{j-1}\right) / m, \quad l\left(\gamma_{l}^{\prime *}\right)>2 j\left(1-a_{j}\right),
$$

where $\gamma_{j}^{\prime}$ is the subcurve of $C_{0}$ that connects the point 1 and the point $a_{j} e^{i \alpha_{j}}$ at which $C_{0}$ meets the circle $\left\{|z|=a_{j}\right\}$ for the last time. Let $\gamma_{j}^{\prime \prime}$ be a subcurve of $\gamma_{j}^{\prime}$ which connects $a_{j} e^{i \alpha_{j}}$ and a point near 1 such that $l\left(\gamma_{j}^{\prime \prime *}\right)>\frac{1}{2} l\left({\gamma^{\prime *}}_{j}\right)$. Now take $b_{j}$ such that

$$
\sup _{z \in \gamma_{j}^{\prime \prime}}|z|<b_{j}<1 .
$$

Let $\gamma_{j}$ be the subcurve of $C_{0}$ that connects $a_{j} e^{i \alpha_{j}}$ and the point $b_{j} e^{i \beta_{j}}$ at which $C_{0}$ meets the circle $\left\{|z|=b_{j}\right\}$ for the first time. Then we see that $0<1-b_{j}<1-a_{j}$ and that $a_{j} \leq|z| \leq b_{j}$ for $z \in \gamma_{j}$. Moreover $\gamma_{j}^{\prime \prime}$ is included in $\gamma_{j}$, so that

$$
l\left(\gamma_{j}^{*}\right)>j\left(1-a_{j}\right) .
$$

Thus we can choose a sequence of subcurves $\gamma_{j}$ with the properties (i)-(iv).

\section{Proof OF THEOREM}

Proof of theorem. For $m=m_{1}$, the constant appearing in Lemma 2, we choose a sequence of subcurves $\gamma_{j}$ as in Lemma 5. For a moment we fix $j$. Let $a_{j}$ and $b_{j}$ be as in Lemma 5. By $M_{j}(\eta)$ we denote the radial line segment $\left\{r e^{i \eta} ; a_{j} \leq\right.$ $\left.r \leq b_{j}\right\}$. We obtain from Lemma 4 applied to $\gamma=\gamma_{j}$ and $M(\eta)=M_{j}(\eta)$ that $T_{\gamma_{j}}\left(M_{j}(\eta)\right)$ is a closed interval of length $l\left(\gamma_{j}^{*}\right)$, where we recall that $T_{\gamma_{j}}$ is the set valued mapping defined before Lemma 4 . Let $N$ be the integer such that

$$
2 \pi / l\left(\gamma_{j}^{*}\right) \leq N<1+2 \pi / l\left(\gamma_{j}^{*}\right)
$$

and let $\eta_{k}=2 \pi k / N$. Since $\left|\eta_{k}-\eta_{k+1}\right| \leq l\left(\gamma_{j}^{*}\right)$, it follows that

$$
[0,2 \pi]=\bigcup_{k=1}^{N} T_{\gamma_{j}}\left(M_{j}\left(\eta_{k}\right)\right)
$$

so that $M_{j}=\bigcup_{k=1}^{N} M_{j}\left(\eta_{k}\right)$ satisfies $T\left(M_{j}\right)=[0,2 \pi]$ by the fact that $T_{\gamma_{j}} z \subset T z$ for $z \in D$. Let

$$
\begin{aligned}
I_{k} & =\left[\eta_{k}-m_{1}\left(1-a_{j}\right), \eta_{k}+m_{1}\left(1-a_{j}\right)\right], \\
E_{j} & =\bigcup_{k=1}^{N} I_{k}, \\
g_{j} & =\chi_{E_{j}} .
\end{aligned}
$$


Secondly, we vary the index $j$ and extract a subsequence of $\gamma_{j}$ satisfying the following (3) and (4). We see from (2) that $\left|E_{j}\right|=2 m_{1}\left(1-a_{j}\right) N \leq$ $2 m_{1}\left(1-a_{j}\right) \cdot 4 \pi / l\left(\gamma_{j}^{*}\right)$, so that $\left|E_{j}\right|$ tends to 0 by (iii) of Lemma 5 . Hence, taking a subsequence of $\gamma_{j}$ if necessary, we may assume that

$$
\sum_{j=1}^{\infty}\left|E_{j}\right|<\infty
$$

We claim that $g_{j}$ satisfies an inequality of type (1). We observe from (2) that an interval of length $4\left(1-b_{j-1}\right)$ in $[0,2 \pi]$ contains at most $\nu$ points $\eta_{k}$, where

$$
\nu \leq 1+\frac{4\left(1-b_{j-1}\right)}{2 \pi / N} \leq \frac{4}{\pi}\left(1-b_{j-1}\right)\left(1+\frac{2 \pi}{l\left(\gamma_{j}^{*}\right)}\right) \leq 16 \frac{1-b_{j-1}}{l\left(\gamma_{j}^{*}\right)} .
$$

The length of $I_{k}$ is equal to $2 m_{1}\left(1-a_{j}\right)<2\left(1-b_{j-1}\right)$ by (iv) of Lemma 5 . Hence if $I$ is an interval of length $2\left(1-b_{j-1}\right)$ in $[0,2 \pi]$, then the number of $I_{k}$ which intersect $I$ is bounded by $16\left(1-b_{j-1}\right) / l\left(\gamma_{j}^{*}\right)$. Therefore $g_{j}$ satisfies

$$
\frac{1}{1-b_{j-1}} \int_{|\varphi-\eta|<1-b_{j-1}} g_{j}(\varphi) d \varphi \leq 32 m_{1} \frac{1-a_{j}}{l\left(\gamma_{j}^{*}\right)}
$$

for all $\eta \in[0,2 \pi]$. Since the right hand side tends to 0 by (iii) of Lemma 5 , it follows from Lemma 3 with $f=g_{j}$ and $c=1-b_{j-1}$ that

$$
\sup _{|z| \leq b_{j-1}} \operatorname{PI}\left(g_{j}, z\right) \leq m_{2} \sqrt{32 m_{1}} \sqrt{\left(1-a_{j}\right) / l\left(\gamma_{j}^{*}\right)}
$$

for sufficiently large $j$. Hence, taking a subsequence of $\gamma_{j}$ if necessary, we may assume that

$$
\sup _{|z| \leq b_{j-1}} \operatorname{PI}\left(g_{j}, z\right) \leq 9^{-j}
$$

Thirdly, we form two sequences $\left\{F_{j}\right\}$ and $\left\{G_{j}\right\}$ of sets inductively. Let $F_{1}=\varnothing, G_{1}=E_{1}$ and let

$$
\begin{aligned}
F_{2 j} & =F_{2 j-1} \cup E_{2 j}, & G_{2 j} & =G_{2 j-1} \backslash E_{2 j}, \\
F_{2 j+1} & =F_{2 j} \backslash E_{2 j+1}, & G_{2 j+1} & =G_{2 j} \cup E_{2 j+1}
\end{aligned}
$$

for $j \geq 1$. We see that $F_{j} \cap G_{j}=\varnothing, F_{j} \cup G_{j}=\bigcup_{k=1}^{j} E_{k}$ and $E_{j} \subset F_{j}$ if $j$ is even; $E_{j} \subset G_{j}$ if $j$ is odd. Let

$$
f_{j}=\left\{\begin{aligned}
1 & \text { on } F_{j}, \\
-1 & \text { on } G_{j}, \\
0 & \text { elsewhere }
\end{aligned}\right.
$$

We observe that if $\varphi \notin \lim \sup E_{j}$, then $f_{j}(\varphi)$ converges to the limit

$$
f(\varphi)=\left\{\begin{aligned}
1 & \text { if } \varphi \in E_{j} \text { with even } j \text { for the last time, } \\
-1 & \text { if } \varphi \in E_{j} \text { with odd } j \text { for the last time, } \\
0 & \text { if } \varphi \notin \bigcup_{j=1}^{\infty} E_{j}
\end{aligned}\right.
$$


It follows from (3) that $\lim \sup E_{j}$ is of measure zero, so that $f_{j}$ converges to $f$ almost everywhere on $[0,2 \pi]$. We note that

$$
\begin{gathered}
f_{j}=(-1)^{j} \quad \text { on } E_{j}, \\
\left|f_{j}\right| \leq 1 \quad \text { on }[0,2 \pi], \\
\left|f_{j}-f_{j+1}\right| \leq 2 g_{j+1} \quad \text { on }[0,2 \pi] .
\end{gathered}
$$

We are now in the final stage. We claim that $h(z)=\operatorname{PI}(f, z)$ is the required bounded harmonic function. It is clear that $|h| \leq 1$ on $D$. Let $h_{j}(z)=$ $\operatorname{PI}\left(f_{j}, z\right)$. Note that $h_{j}$ converges to $h$ uniformly on every compact set in $D$ by the dominated convergence theorem. We infer from (5), (6) and Lemma 2 with $f=f_{j}, \eta=\eta_{k}$ and $c=1-a_{j}$ that if $j$ is even, then

$$
h_{j}(z) \geq \frac{1}{2} \quad \text { for } z \in M_{j}
$$

if $j$ is odd, then

$$
h_{j}(z) \leq-\frac{1}{2} \quad \text { for } z \in M_{j} .
$$

We infer from (4) and (7) that

$$
\sup _{|z| \leq b_{j}}\left|h_{j}(z)-h_{j+1}(z)\right| \leq 2 \sup _{|z| \leq b_{j}} \operatorname{PI}\left(g_{j+1}, z\right) \leq 2 / 9^{j+1} \text {. }
$$

Hence

$$
\sum_{k=j}^{\infty} \sup _{|z| \leq b_{j}}\left|h_{k}(z)-h_{k+1}(z)\right| \leq \sum_{k=j}^{\infty} \frac{2}{9^{k+1}} \leq \frac{1}{4}
$$

Therefore, if $j$ is even, then

$$
h(z)=h_{j}(z)+\left(h_{j+1}(z)-h_{j}(z)\right)+\cdots \geq \frac{1}{2}-\frac{1}{4}=\frac{1}{4} \quad \text { for } z \in M_{j} ;
$$

if $j$ is odd, then

$$
h(z) \leq-\frac{1}{4} \quad \text { for } z \in M_{j} .
$$

Since $T\left(M_{j}\right)=[0,2 \pi]$, it follows that if $j$ is even, then

$$
\sup _{a_{j} \leq|z| \leq b_{j}, z \in C_{\theta}} h(z) \geq \frac{1}{4} \quad \text { for all } \theta \in[0,2 \pi]
$$

if $j$ is odd, then

$$
\inf _{a_{j} \leq|z| \leq b_{j}, z \in C_{\theta}} h(z) \leq-\frac{1}{4} \quad \text { for all } \theta \in[0,2 \pi] .
$$

Consequently,

$$
\liminf _{|z| \rightarrow 1, z \in C_{\theta}} h(z) \leq-\frac{1}{4}<\frac{1}{4} \leq \limsup _{|z| \rightarrow 1, z \in C_{\theta}} h(z) \quad \text { for all } \theta \in[0,2 \pi] .
$$

Thus this $h$ satisfies the required property. The proof is complete.

Remark. Let $E_{j}$ be as in the above proof. Since $\left|E_{j}\right| \rightarrow 0$, we can take a nonnegative sequence $\left\{p_{j}\right\}$ such that $\lim \sup _{j \rightarrow \infty} p_{j}=\infty$ and $f=\sum_{j=1}^{\infty} p_{j} \chi_{E_{j}}$ is integrable. In view of Lemma 2 and $T\left(M_{j}\right)=[0,2 \pi]$, we obtain that $h=$ $\operatorname{PI}(f, z)$ is a positive harmonic function such that

$$
\limsup _{|z| \rightarrow 1, z \in C_{\theta}} h(z)=\infty \quad \text { for all } \theta \in[0,2 \pi]
$$




\section{REFERENCES}

1. D. A. Brannan and J. G. Clunie, Aspects of contemporary complex analysis, Academic Press, London, 1980.

2. F. E. Collingwood and A. J. Lohwater, The theory of cluster sets, Cambridge University Press, London, 1966.

3. J. E. Littlewood, On a theorem of Fatou, J. London Math. Soc. 2 (1927), 172-176.

4. A. J. Lohwater and G. Piranian, The boundary behavior of functions analytic in a disk, Ann. Acad. Sci. Fenn. Ser. A I, no. 239 (1957).

Department of Mathematics, Faculty of Engineering, Gunma University, Kiryu 376, JAPAN 